\title{
Analysis of geochemical basalts database as a simulant for uranium ore concentrates data: the Galaxy Serpent 3 Exercise
}

\author{
Itzhak Sedgi ${ }^{1} \cdot$ Maor Assulin ${ }^{1} \cdot$ Amiram Moyal ${ }^{1} \cdot$ Boaz Astarchan $^{1} \cdot$ Arnon Rubinshtein $^{1} \cdot$ Eyal Elish $^{1}$ (I)
}

(c) Springer Nature Switzerland AG 2019

\begin{abstract}
The Nuclear Forensics International Technical Working Group (NF-ITWG) conducted an exercise, Galaxy Serpent 3 (GS3), by the National Nuclear Forensics Libraries Task Group, during 2017. The main goal of the GS3 exercise was to illustrate the usefulness of a National Nuclear Forensic Library for assessing the potential consistency of unknown material with national holdings. Moreover, it offers the possibility to produce a realistic database resembling uranium ore concentrate (UOC) that can be used for algorithm development activities. A database was built based on basalt compositions from different tectonic settings: MORB, IAB, OIB and CRFB. Classes (Affinities) with subtly different chemical compositions. All data are open source and sample names can be traced to original data for error checking. Participants (29 teams) had to use existing techniques, or develop their own algorithms, to allow for dealing with the sparse database and managing incomplete data points. The exercise's scenario introduced 3 simulated origin-unknown UOC samples, interdicted by authorities. Each team was asked to answer two main questions-"Are the 3 unknown samples consistent with each other?" and "are the 3 unknown samples consistent with the Mines in the database given?". For the first time, the Israeli National Nuclear Forensic Laboratory (INNFL) participated in the GS exercise. A dedicated team was assigned the task of building capabilities needed and applying them to answer the raised questions. Methodology and tools developed and applied by the Israeli team are described. The results are presented and discussed, including new signatures allowing differentiation between unknown samples and mines.
\end{abstract}

Keywords Nuclear forensics · National Nuclear Forensics Libraries · Principle component analysis · Ratio correlation matrix

\section{Introduction}

Data analytics is essential for analyses of large data sets emerging during a nuclear forensic investigation [1]. When measured results, from various analytical techniques [1-4], are gathered, precise answers to the questions of origin and route of materials involved are required [5-7]. The answers to these fundamental questions are mostly not clear since there is always a variance between samples, and even between identical samples (due to the uncertainty in the analytical measurement). Moreover, geographical and geochemical parameters affect the composition of these samples as well [8,9], resulting from composition differences that, at first glance, seem totally unrelated to the sample.

Galaxy serpent is a virtual tabletop exercise intended to enhance the National Nuclear Forensics Library (NNFL) capabilities of the participants. Exercises are conducted under the Nuclear Forensics International Technical Working Group (ITWG) and organized by the NNFL task group [10]. The third exercise was held during 2017 and involved a synthetic database, resembling a uranium ore concentrate (UOC) database, which was intentionally designed to match a realistic database in the sense of missing data and errors. The database contained 821 samples which have been divided into 4 Mines- IAB, MORB, OIB and CRFB. The

Eyal Elish, eyalel@nrcn.gov.il | ${ }^{1}$ Nuclear Research Center Negev (NRCN), P.O.B 9001, 8419001 Beer Sheva, Israel.

SN Applied Sciences (2019) 1:806 | https://doi.org/10.1007/s42452-019-0831-7 
database contained elemental concentrations only, without any isotopic information. The exercise scenario introduced three UOC samples of unknown origin that have been seized by authorities with the following sample ID's: 1005, 8615-OSF-1 and 53023. Each team has been asked to answer two main questions-" "Are the 3 unknown samples consistent with each other?" and, "are the 3 unknown samples consistent with the Mines in the database given?"

\section{Classification methods}

To classify the unknown samples with high confidence, several different methods were applied. Three methods utilize the rare-earth elements (REE) concentrations as the basis for answering the questions asked. The REE pattern normalized to chondrite [11] has been successfully used for origin tracing $[12,13]$. The REE pattern has recently been found to be a useful signature even to processed materials [14]. Another method presented, not related to REE data, was based on the correlations between the element ratios in the different group of materials.

\subsection{REE signature normalized to chondrite}

The REEs share similar chemical properties [15], and when normalized to chondrite, a distinguished-mine pattern emerges. This signature can be used to trace the origin of UOC samples [16]. Figure 1 presents the relative concentration of REE/chondrite on a logarithmic scale for each mine in the database. Each graph represents the average value of a specific mine. AIB is easily distinguished from the rest of the mines, forming a "V" shape, related to the Eu-anomaly [17]. OIB shows a monotonically decreasing pattern, while CRFB shows an increasing pattern. MORB shows a flat profile; however, it is not ambiguously separable from CRFB when plotting each of the samples separately, and not using the averaged values. Attribution using REE pattern of uranium deposits can be carried out considering the distinguished deposit profile. The subjected basalt database shows similarities with some of the uranium deposit REE pattern [12] and straightens the resemblances of the chosen basalts to uranium deposits.

The chondrite-normalized REE pattern of the three unknown samples is presented in Fig. 2. The unknown samples 53023 and 8615 -OSF-1 are showing the same pattern as IAB, while 1005 shows a similar pattern to OIB.

\subsection{Principle component analysis (PCA) for the REE}

The REEs are highly correlated with each other, and their correlation may resemble an information redundancy which may be revealed, using principle component analysis [18-20]. At this point, due to considerable lack of REEs composition data, and lower priority to develop algorithms for overcoming it, we decided to concentrate on developing methods to strengthen analysis of existing data. The PCA calculations were conducted after database pretreatment which included centering and normalization. Only the samples in the database which presented missing data in their REE section were ignored. The prediction capabilities of this method have been examined by excluding $10 \%$ of the database and using it as test samples. All the test results were qualitatively accurate. Figure 3 shows the projection of the database samples and the unknown samples, on the main two components. A total of $90 \%$ of the starting information is saved using only the first 2 principal components.
Fig. 1 Chondrite-normalized REE pattern of the different mines presented in the database

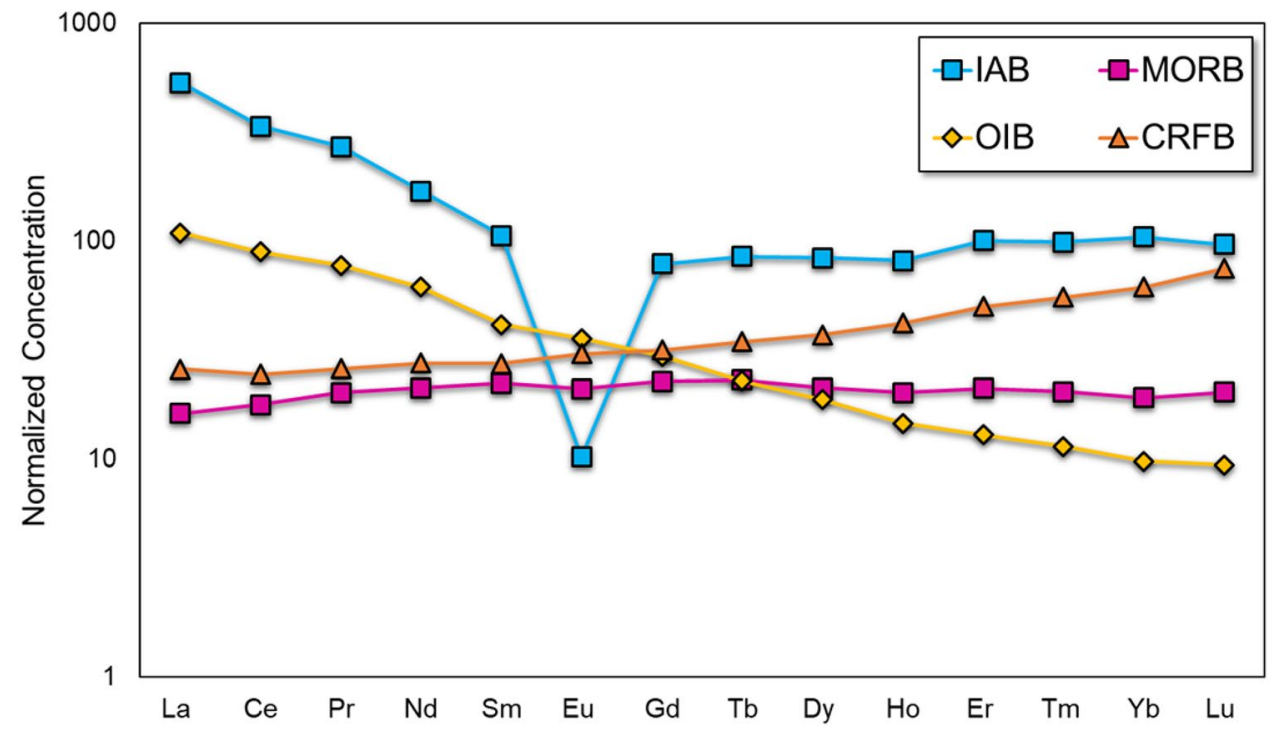


Fig. 2 Chondrite-normalized REE pattern of the 3 unknown samples
Fig. 3 Principal component analysis of the REE for the mines in the database and for the unknown samples (glow)
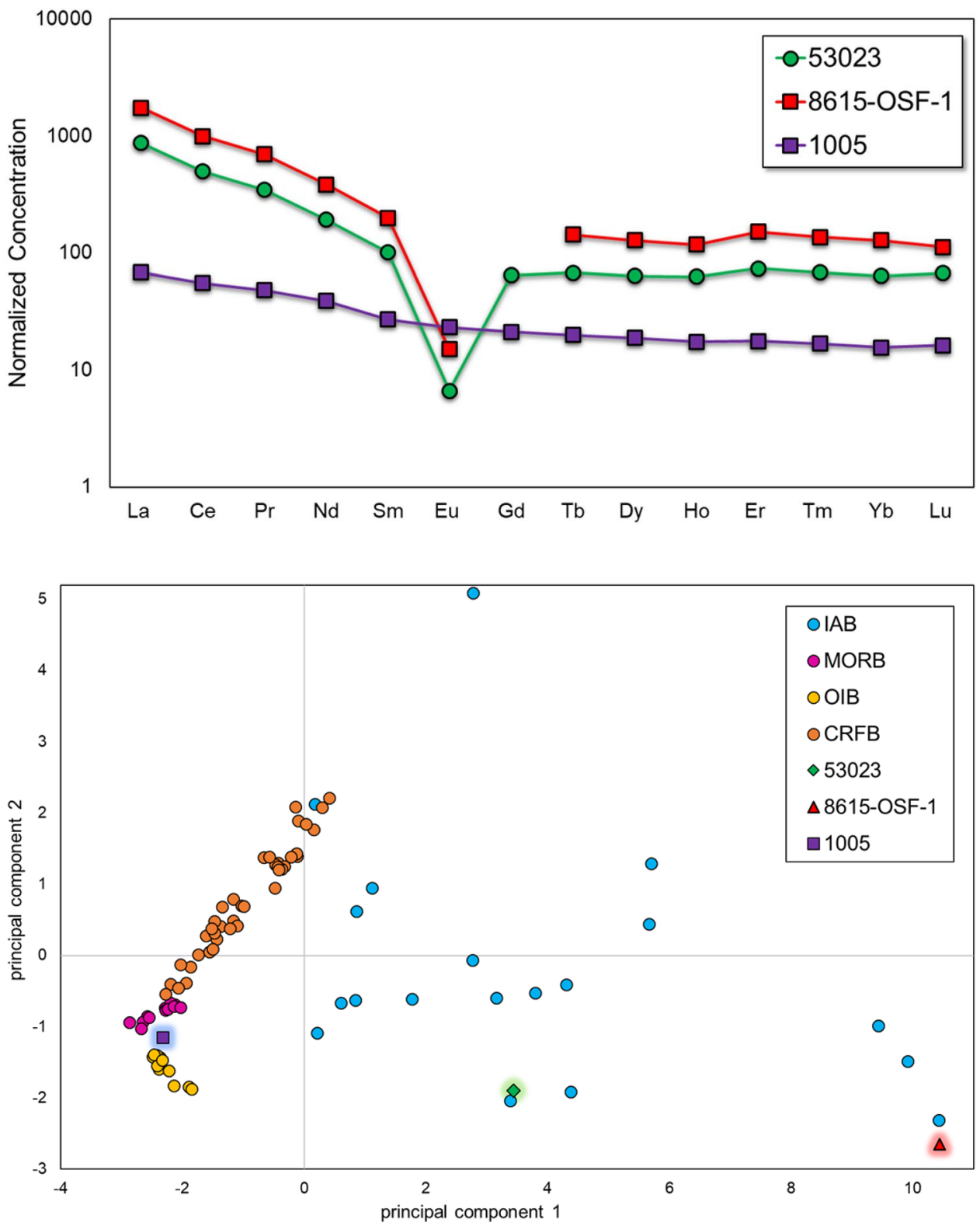

According to Fig. 3, the unknown samples 53023 and 8615-OSF-1 are part of the IAB group, while 1005 is not related, seemingly, to any mine group. This result strengthens our confidence that 53023 and 8615 -OSF- 1 can be associated with the same type of deposit, hence also with IAB mine. In contrast to that, the REE data of sample 1005 appear no to be consistent with that of the IAB mine and are not related to the other two unknown samples.

\subsection{REE ratios comparison}

To produce some quantitative information using the REE concentrations, we compared all the possible REE ratios in a single sample, to all the other REE ratios in all the other samples. As an example, for a sample which contains information for 13 elements, there are 78 different ratio possibilities which can be used to build a 78 -element vector. Building in the same way 78 ratios vector for each sample in the database and compare each fitted element in the sample vector to every other vector in the database while assigning a weight of $1 / 78$, will produce a factor between 0 and 100 for each examined pair, were 100 means identical samples. Figure 4 presents the calculated factor of each unknown sample to the relevant samples in the database. The prediction capabilities of this method have also been examined by excluding $10 \%$ of the database and using it as a test. All tests results produced a high factor value $(\sim 90)$ to their parent mine indicating a successful prediction. 


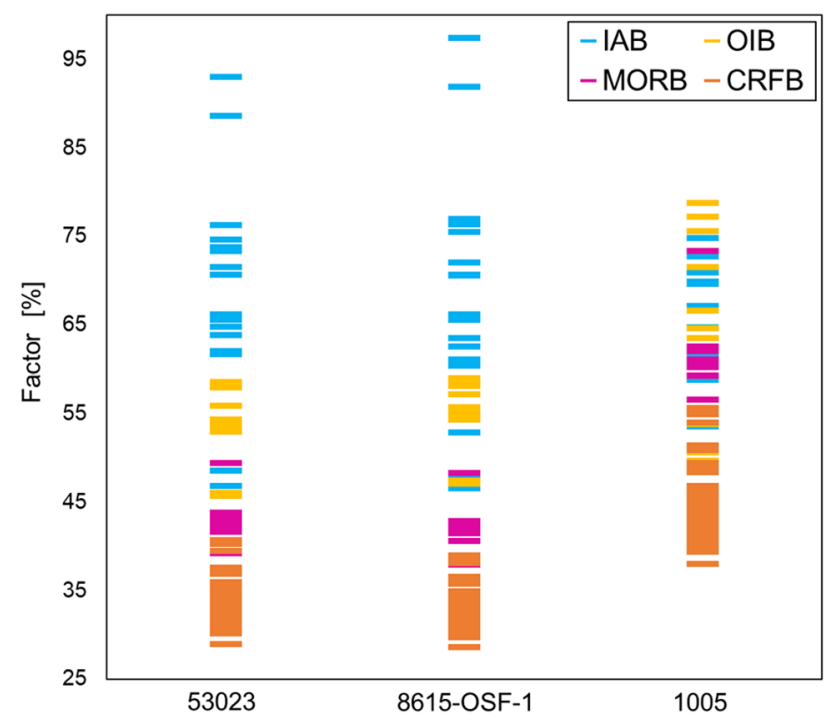

Fig. 4 REE ratios similarities between the unknown samples and the database samples. Each vertical line is a sample from the database. High factor values mean a higher similarity to the examined sample

Figure 4 shows that unknown samples 53023 and 8615-OSF-1 yielded a high factor value to samples from the AIB mine. This indicates that those two unknown samples originate from the AIB mine. The unknown sample 1005, shows much lower value and disperse factor results to all the samples in the database the closest mine to 1005 is OIB, but it is much less distinct.

\subsection{Correlations: revealing hidden signatures}

To reveal signatures which are not exclusively dependent on the REE, correlations between elements and between the element ratios were searched. The elements correlation revealed a significant connection between the REEs and since a broad analysis has been conducted using REE exclusively, only the elements ratios were used further. The main effort was given to ratios that will present the most significant difference between AIB and OIB mines. The likelihood of 53023 and 8615 -OSF-1 being part of the AIB mine and the none zero possibility of 1005 being a part of the OIB mine emphasize this choice. The search for the most significant difference has been done by comparing the correlation coefficients of all the possible ratios between the mines and extracting the ones showing the highest difference. After building a matrix (or vector) of all the possible elements ratios, which in this case contained tens of thousands of ratios, a correlation matrix between the ratios has been built. A high absolute correlation coefficient indicates a better correlation. By conducting the same procedure for each mine separately, differences between the correlation coefficient of specific elements ratio can be found and revealed good basis for separation. By plotting elements ratio which has high correlation coefficients in one mine and small correlation coefficient in the other was found to be very useful. To illustrate the power of this method, two results are presented in Fig. 5. The figure shows the $\mathrm{Mn} / \mathrm{Ti}$ ratio relative to the $\mathrm{Mn} / \mathrm{Mg}$ ratio. The AIB group is clearly different from the OIB group, and the two unknown samples, 53023 and 8615-OSF-1, are part of
Fig. $5 \mathrm{Mn} / \mathrm{Ti}$ ratio relative to $\mathrm{Mn} / \mathrm{Mg}$ ratio, for IAB samples, OIB samples and the unknown samples (glow)

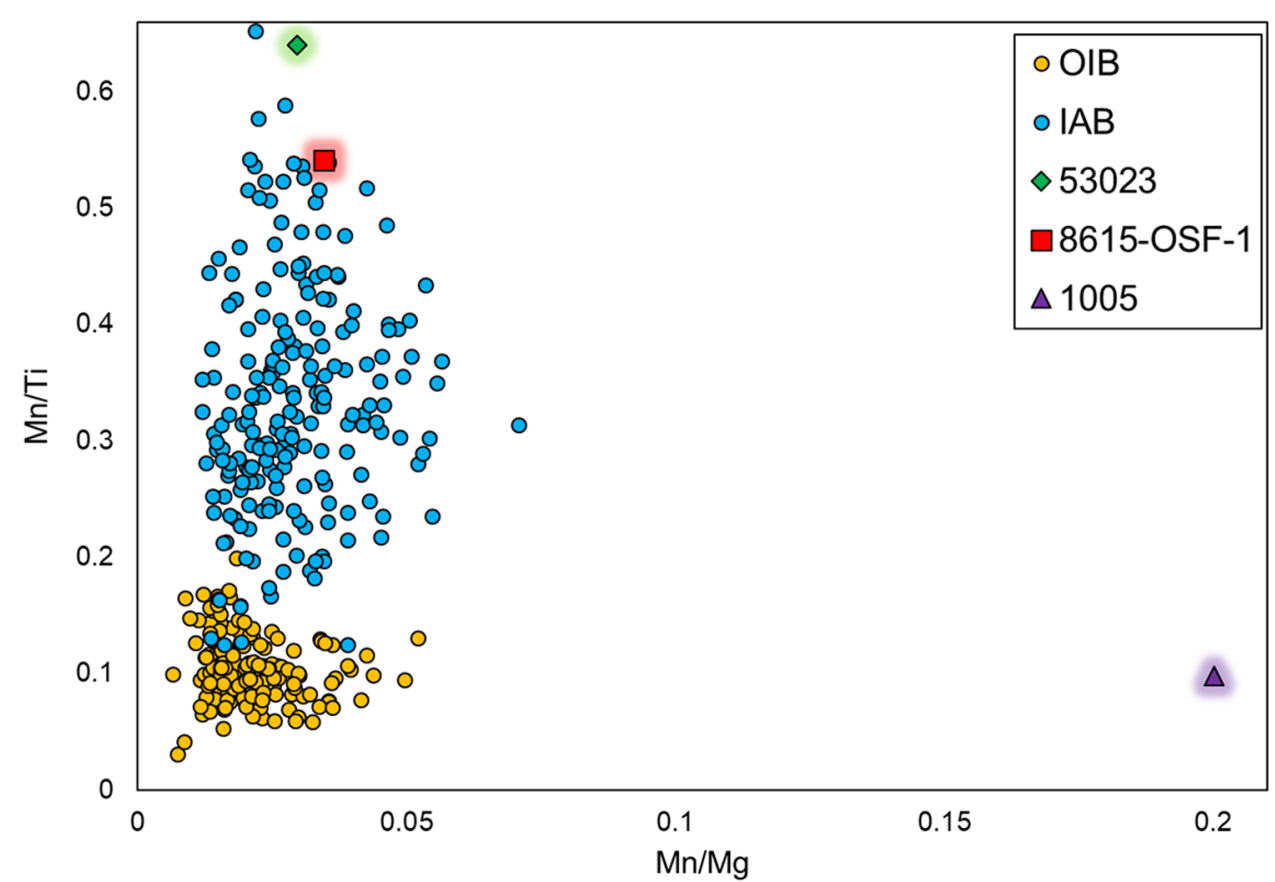


the AIB group. The unknown sample 1005 is significantly different from the OIB and AIB groups altogether.

Figure 6 shows the Th/Fe ratio relative to the Th/Ca ratio. These two ratios reveal a high correlation in the AIB mine samples with a significant difference between the AIB and the OIB mines. The unknown sample 53023 is part of the straight line dots representing the AIB mine. The $8615-O S F-1$ is not presented due to missing data, and 1005 is not seen in the figure due to a scale difference. 1005 has two orders of magnitude difference in its coordinate $(33,30)$ from all the rest of the data. Figures 5 and 6 are showing two signatures which clearly indicate the consistency of 53023 and 8615-OSF-1 with data from AIB. In contrast to that, sample 1005 appears not to be consistent with the AIB or the OIB mines.

\section{Discussion}

Statistical methods that were applied for analysis of the database are indifferent to the origin or geochemistry of the samples. The procedures developed are based on mathematical principles and hence can be applied to either synthetic or real database. Relations between different elements, as reviled after applying the algorithms, truly represent actual differences, in the "real world" which can serve as signatures to distinguish between samples. The procedure developed might expose hidden signatures when applied on actual data gathered from analysis of materials like UOCs. The decision to simulate UOCs database by using basalts proved to be appropriate, as can be seen considering the results of REEs pattern (such as Euanomaly) as compared to the known pattern published in the literature.

\section{Conclusions}

The REE/chondrite signature has proven to be the simplest, yet most effective tool for fast qualitative reference starting point analysis. The consistency of the unknown samples 53023 and 8615-OSF-1 with AIB was apparent due to the Eu-anomaly in the AIB mine. The REE ratio comparison has been used as enforcement of the REE/chondrite result. In addition, using PCA to the REE revealed a more precise separation between the mines and straightened the confidence in the origin of 53023 and 8615-OSF-1. Then, the correlation analysis presented served as a very powerful tool to reveal differences between various sets of data, and by choosing the right elements for separation, a significant result was achieved due to the consistency of the unknown samples with the database.

The methods presented in this work indicate that the unknown samples 53023 and 8615-OSF-1 are consistent with each other and originated from the mine in the database referred to as AIB. The unknown sample 1005 is not consistent with the other two unknown samples, and
Fig. $6 \mathrm{Th} /$ Fe ratio relative to Th/Ca ratio, for IAB samples, OIB samples and the unknown samples 53023 and 1005 (glow). 1005 is out of scale, with coordinate $(33,30)$

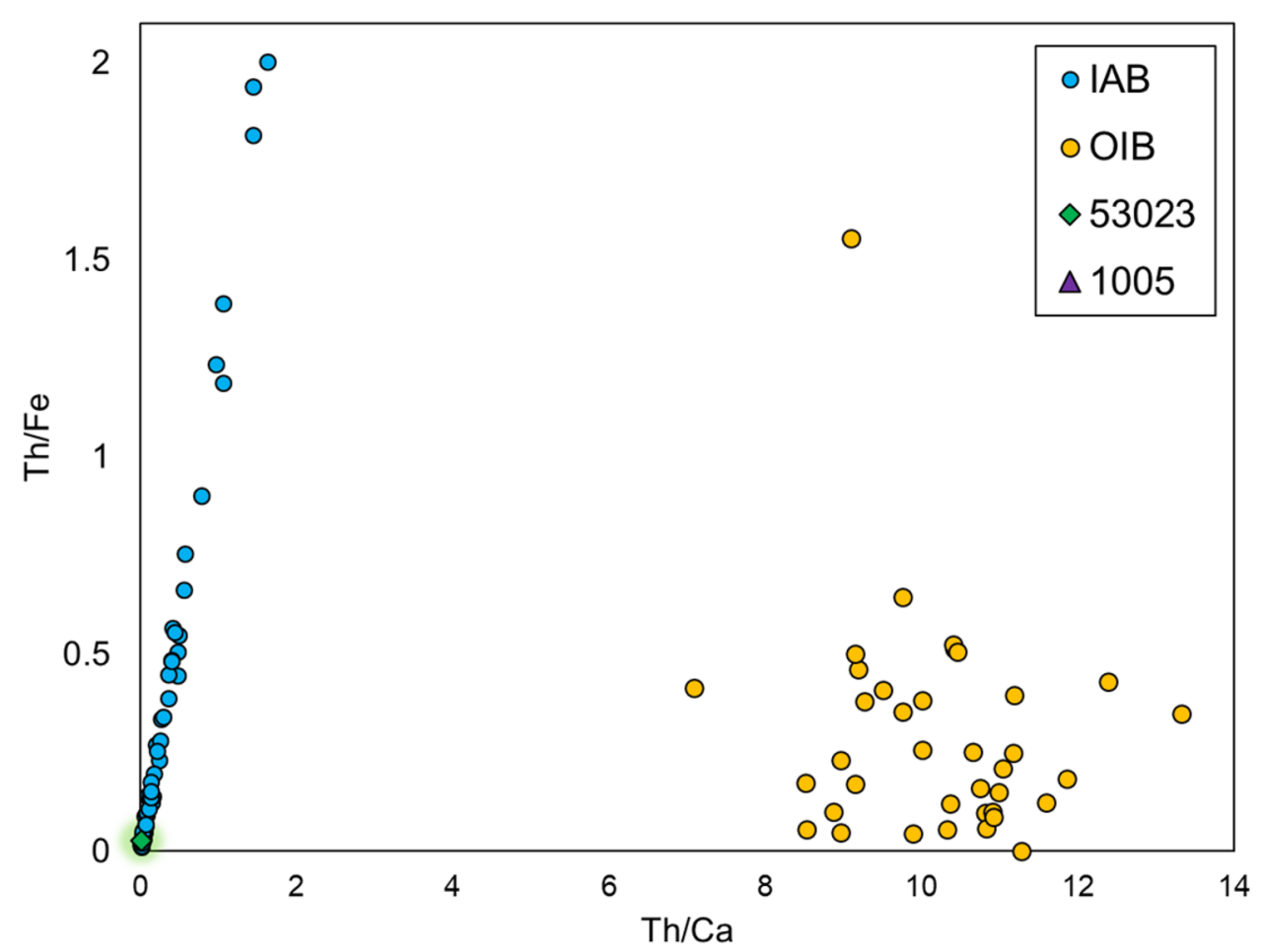

SN Applied Sciences 
furthermore, it is not consistent with neither of the mines in the database.

The results of GS3 were presented by the exercise designers during the 23rd meeting of the Nuclear Forensics ITWG at Fribourg Switzerland. Our conclusions, as presented above, matched precisely the official results and proved the validation of the data analysis techniques applied.

The Israel National Nuclear Forensic Laboratory (INNFL) participated in the Galaxy Serpent exercise for the first time and used the opportunity to improve data analysis capabilities. Moreover, different methods were applied and comparatively discussed. The exercise derived our team to enhance our capabilities in the field of data analytics, and we will further expand the knowledge needed to develop new tools.

\section{Compliance with ethical standards}

Conflict of interest On behalf of all authors, the corresponding author states that there is no conflict of interest.

\section{References}

1. Kristo MJ (2012) Nuclear forensics. In: L'Annunziata MF (ed) Handbook of radioactivity analysis, 3rd edn. Academic Press, Amsterdam, pp 1281-1304

2. Stanley FE, Stalcup AM, Spitz HB (2013) A brief introduction to analytical methods in nuclear forensics. J Radioanal Nucl Chem 295(2):1385-1393

3. Mayer K, Wallenius M, Varga Z (2013) Nuclear forensic science: correlating measurable material parameters to the history of nuclear material. Chem Rev 113(2):884-900

4. Keegan E, Kristo MJ, Toole K, Kips R, Young E (2016) Nuclear forensics: scientific analysis supporting law enforcement and nuclear security investigations. Anal Chem 88(3):1496-1505

5. El Haddad J et al (2018) Discrimination of uranium ore concentrates by chemometric data analysis to support provenance assessment for nuclear forensics applications. J Radioanal Nucl Chem 317(1):625-632
6. Reading DG, Croudace IW, Warwick PE, Cigliana KA (2016) Applying multivariate statistics to discriminate uranium ore concentrate geolocations using (radio)chemical data in support of nuclear forensic investigations. J Environ Radioact 162-163:172-181

7. Mayer K, Wallenius M, Fanghänel T (2007) Nuclear forensic science-from cradle to maturity. J Alloys Compd 444-445:50-56

8. Spano TL et al (2017) A novel nuclear forensic tool involving deposit type normalized rare earth element signatures. Terra Nova 29(5):294-305

9. Spano TL, Simonetti A, Balboni E, Dorais C, Burns PC (2017) Trace element and $U$ isotope analysis of uraninite and ore concentrate: applications for nuclear forensic investigations. Appl Geochem 84:277-285

10. Borgardt J, Wong F (2014) Galaxy Serpent: a web-based tabletop exercise using the concept of National Nuclear Forensics Libraries. J Nucl Mater Manag Summer 67(4):4-11

11. Anders $E$, Nicolas $G$ (1989) Abundances of the elements: meteoritic and solar. Geochim Cosmochim Acta 53:197-214

12. Mercadier J et al (2011) Origin of uranium deposits revealed by their rare earth element signature. Terra Nova 23(4):264-269

13. Varga Z, Wallenius M, Mayer K (2010) Origin assessment of uranium ore concentrates based on their rare-earth elemental impurity pattern. Radiochim Acta Int J Chem Asp Nucl Sci Technol 98(12):771-778

14. Varga $Z$ et al (2017) Identification of uranium signatures relevant for nuclear safeguards and forensics. J Radioanal Nucl Chem 312(3):639-654

15. Moody KJ, Grant PM, Hutcheon ID (2014) Nuclear forensic analysis, 2nd edn. CRC Press, Boca Raton

16. Fedchenko V (ed) (2015) The new nuclear forensics: analysis of nuclear materials for security purposes. Oxford University Press, Oxford

17. Weill DF, Drake MJ (1973) Europium anomaly in plagioclase feldspar: experimental results and semiquantitative model. Science 180(4090):1059-1060

18. Bro R, Smilde AK (2014) Principal component analysis. Anal Methods 6(9):2812-2831

19. Brereton RG (2009) Chemometrics for pattern recognition. Wiley, Chichester

20. Bishop C (2006) Pattern recognition and machine learning. Springer, New York

Publisher's Note Springer Nature remains neutral with regard to jurisdictional claims in published maps and institutional affiliations. 